\title{
An estimation method of the direct benefit of a waterlogging control project applicable to the changing environment
}

\author{
LIU ZENGMEI ${ }^{1,2}$, QIN GUANGHUA ${ }^{2}$ \& CHEN ZISHEN ${ }^{3}$ \\ 1 Department of Water Conservancy and Hydropower Engineering, South China University of Technology, Guangzhou, \\ 510640, China \\ liuzm@scut.edu.cn \\ 2 State Key Laboratory of Hydraulics \& Mountain River Engineering, Sichuan University, 610065, China \\ 3 Department of Water Resources \& Environmental Engineering, Zhongshan University, Guangzhou, 510275, China
}

\begin{abstract}
The direct benefit of a waterlogging control project is reflected by the reduction or avoidance of waterlogging loss. Before and after the construction of a waterlogging control project, the disaster-inducing environment in the waterlogging-prone zone is generally different. In addition, the category, quantity and spatial distribution of the disaster-bearing bodies are also changed more or less. Therefore, under the changing environment, the direct benefit of a waterlogging control project should be the reduction of waterlogging losses compared to conditions with no control project. Moreover, the waterlogging losses with or without the project should be the mathematical expectations of the waterlogging losses when rainstorms of all frequencies meet various water levels in the drainage-accepting zone. So an estimation model of the direct benefit of waterlogging control is proposed. Firstly, on the basis of a Copula function, the joint distribution of the rainstorms and the water levels are established, so as to obtain their joint probability density function. Secondly, according to the two-dimensional joint probability density distribution, the dimensional domain of integration is determined, which is then divided into small domains so as to calculate the probability for each of the small domains and the difference between the average waterlogging loss with and without a waterlogging control project, called the regional benefit of waterlogging control project, under the condition that rainstorms in the waterlogging-prone zone meet the water level in the drainage-accepting zone. Finally, it calculates the weighted mean of the project benefit of all small domains, with probability as the weight, and gets the benefit of the waterlogging control project. Taking the estimation of benefit of a waterlogging control project in Yangshan County, Guangdong Province, as an example, the paper briefly explains the procedures in waterlogging control project benefit estimation. The results show that the waterlogging control benefit estimation model constructed is applicable to the changing conditions that occur in both the disaster-inducing environment of the waterlogging-prone zone and disaster-bearing bodies, considering all conditions when rainstorms of all frequencies meet different water levels in the drainageaccepting zone. Thus, the estimation method of waterlogging control benefit can reflect the actual situation more objectively, and offer a scientific basis for rational decision-making for waterlogging control projects.
\end{abstract}

Key words changing environment; waterlogging control benefit; waterlogging loss; estimation; mathematical expectation; copula function; joint probability density function

\section{BACKGROUND}

The term waterlogging disaster refers to a condition such that, due to excessive rainfall, surface rainwater cannot be immediately removed, resulting in surface ponding, which immerses the disaster-bearing body, so that damages are caused to the functions and values of the disasterbearing body, and cause losses to the economy. Obviously, the degree of ultimate disaster is closely related to the condition of water accumulation and the capability of the disaster-bearing body to withstand disasters. The condition of water accumulation caused by the same rainstorm is not only related to the disaster-inducing environment in a waterlogging-prone zone (referring to the condition of the underlying surface, water storage capacity, drainage systems, etc.), but also closely related to the water level in the drainage-accepting zone. Therefore, for the same rainstorm, any change in the factors of the disaster-bearing body or disaster-inducing environment of the waterlogging-prone zone or water level in the drainage-accepting zone may lead to a corresponding change in the degree of disaster, and the waterlogging loss will change along with it.

The disaster-inducing environment of a waterlogging-prone zone may change over time, and the type, quantity and spatial distribution of a disaster-bearing body may also change over time. For instance, with urbanisation, the changes of underlying surface lead to changes in the conditions of runoff generation and flow concentration. Apart from this, urbanization is often 
accompanied by industrial structure adjustment and the disaster-bearing body is rapidly changing, so the total economic value and the disaster-bearing capacity will certainly change accordingly.

In addition, the drainage-accepting zone may be a river, lake or sea, of which the water level generally changes, especially when the drainage-accepting zone is a tidal river or sea. The water level has an influence over the draining-off of water from water-logged zone.

The direct economic benefit of a waterlogging control project is the loss that can be reduced or avoided by the project, which is the difference between the loss suffered without the project and the loss suffered with the project already in operation. Therefore, under the changing environment, the benefit of a waterlogging control project should be the difference between the waterlogging losses with and without the waterlogging control project. And the waterlogging loss, no matter with or without the waterlogging control project, should be the mathematical expectation value of waterlogging loss under all possible combinations of rainstorms in the waterlog-prone zone and water levels in the drainage-accepting zone. The reason is that a rainstorm, no matter what frequency it has, may meet various water levels in the drainage-accepting zone.

Methods commonly used for waterlogging control benefits estimation, such as the measurement of accumulated waterlogging, the correlation analysis method of axis, the actual annual series method (Shi Xichan and Jiang Shuixin 2005, Wang Li-ping et al. 2008, Zhang Dazhi. 2004) are currently not suitable for the assessment of waterlogging control benefits when the environment is changing. Up till now, there were few studies on the estimation of waterlogging control benefits (Guo qi. (2002), Li Jiren et al. 2003, Zhu Xuping et al. 2007), which never provide an actual method to estimate waterlogging control benefits. With the rapid development of urbanization and economic society, waterlogging disasters have become more and more serious. It is urgent to strengthen the waterlogging control, and thus it is critical to develop new methods to estimate waterlogging control benefits under the constantly changing environment. The key of the study is to investigate how to predict waterlogging control benefits in any year.

The estimation model of the direct benefit of waterlogging control projects put forward in this paper may be suitable for the changes of both disaster-inducing environment and disaster-bearing bodies. In addition, it considers all the combinations between rainstorms of the waterlog-prone zone and water levels in the drainage-accepting zone. Therefore, the proposed estimation method of waterlogging control benefits can objectively reflect the actual benefits. Taking the estimation of the benefit of a waterlogging control project in Yangshan County, Guangdong Province as an example, the paper briefly explains the procedures in waterlogging control project benefit estimation.

\section{THE WATERLOGGING CONTROL BENEFITS ESTIMATION MODEL}

\subsection{The construction of the estimation model of waterlogging control benefits}

Both the rainstorm in a waterlog-prone zone, $H$, and the water level encountered in a drainageaccepting zone, $Z$, are continuous random variables. For any possible combination of $h$ and $z$, referred to as $(h, z)$, both the waterlogging loss without a waterlogging control project, $S_{0}$, and the loss with a control project, $S_{1}$, are functions of $h$. Assuming that $S_{j}=g_{j}(h, z)(j=0,1)$, where $g_{0}(h, z)$ and $g_{l}(h, z)$ are the waterlogging loss functions with or without a waterlogging control project, respectively. Then, $S_{j}$, i.e. $S_{0}$ and $S_{1}$, is also a random variable. The waterlogging loss with or without a control project can be generally represented as $E\left(S_{j}\right)(j=0,1)$. Assuming that the probability density of two-dimensional random variable $(H, Z)$ related to the point $(h, z)$, i.e. the combination of $h$ and $z$, is $f(h, z)$. Then:

$$
E\left(S_{j}\right)=E\left[g_{j}(h, z)\right]=\int_{0}^{+\infty} \int_{z_{0}}^{+\infty} g_{j}(h, z) f(h, z) \mathrm{d} h \mathrm{~d} z=\iint_{D} g_{j}(h, z) f(h, z) \mathrm{d} h \mathrm{~d} z
$$

where $D$ is the integral region, $D: 0 \leq h<+\infty, z_{0} \leq z<+\infty$. $z_{0}$ is the possible minimal water level in a drainage-accepting zone. The integral region can be further determined by combining $H$ (the rainstorm in a waterlog-prone zone) and $Z$ (the corresponding water level in a drainage-accepting zone) with the marginal probability density distribution $f_{H}(h)$ and $f_{Z}(z)$.

Therefore, the waterlogging control benefit, $B$, is: 


$$
B=E\left(S_{\mathrm{o}}\right)-E\left(S_{1}\right)=\iint_{D}\left[g_{\mathrm{o}}(h, z)-g_{1}(h, z)\right] f(h, z) \mathrm{d} h \mathrm{~d} z
$$

where $f(h, z)$ is the probability density of $H$ and $Z$ at the point $(h, z)$, i.e. the combination of $h$ and $z$.

$f(h, z)$ can be obtained according to the methods introduced by Liu Zengmei and Chen Zhishen. (2009, 2011). This makes it possible to apply the method of waterlogging control benefits calculation mentioned above. However, it is still difficult to use equation (1) to directly estimate the expectation value of waterlogging loss with or without a control project. First of all, $S_{j}=$ $g_{j}(h, z)(j=0,1)$ is generally not available. Secondly, two integrals may not be given by the elementary function. Hence, it is necessary to develop practical calculation methods.

Here, the widely used "segmentation-approximate-integration" method in engineering mathematics is applied to approximately solve equation (1). Its calculation precision is closely related to how fine the segmentation of the integral region $D$ is. The finer, the accuracy of the results is higher.

Two families of straight lines, which are respectively parallel with two axes and have individual integral distance of $\Delta h$ and $\Delta z$, divide the integral region $D$ into many small closed areas $\Delta \sigma_{i}(i=1,2 \ldots, n)$ (Fig. 1). $\Delta h$ and $\Delta z$ do not need to be fixed values. Then:

$$
E\left(S_{j}\right)=E\left[g_{j}(h, z)\right]=\iint_{D} g_{j}(h, z) f(h, z) \mathrm{d} h \mathrm{~d} z \approx \sum_{i=1}^{n} \iint_{\Delta \sigma_{i}} g_{j}(h, z) f(h, z) \mathrm{d} h \mathrm{~d} z
$$

The average waterlogging loss $\overline{S_{j i}}$ at $\Delta \sigma_{i}$ is expressed as $(j=0,1 ; i=1,2 \ldots \ldots n) . \iint_{\Delta \sigma_{i}} f(h, z) \mathrm{d} h \mathrm{~d} z$ is the probability, $\Delta p_{i}$, of the point $(h, z)$ at $\Delta \sigma_{i}$. The approximate calculation equation is obtained by equation (4).

$$
E\left(S_{j}\right)=E\left[g_{j}(h, z)\right] \approx \sum_{i=1}^{n} \iint_{\Delta \sigma_{i}} g_{j}(h, z) f(h, z) \mathrm{d} h \mathrm{~d} z \approx \sum_{i=1}^{n} \overline{s_{j i}} \iint_{\Delta \sigma_{i}} f(h, z) \mathrm{d} h \mathrm{~d} z=\sum_{i=1}^{n} \overline{s_{j i}} \Delta p_{i}
$$

Hence, the equation of waterlogging control project benefits is as follows:

$$
B=E\left(S_{0}\right)-E\left(S_{1}\right) \approx \sum_{i=1}^{n} \overline{s_{0 i}} \Delta p_{i}-\sum_{i=1}^{n} \overline{s_{1 i}} \Delta p_{i}=\sum_{i=1}^{n}\left(\overline{s_{0 i}}-\overline{s_{1 i}}\right) \Delta p_{i}=\sum_{i=1}^{n} \Delta B_{i} \Delta p_{i}
$$

Equation (5) shows that the probability is used as weight to perform the weighted average for the waterlogging control benefits of all small rectangular regions. Its average is approximate to the waterlogging control benefit of the project.

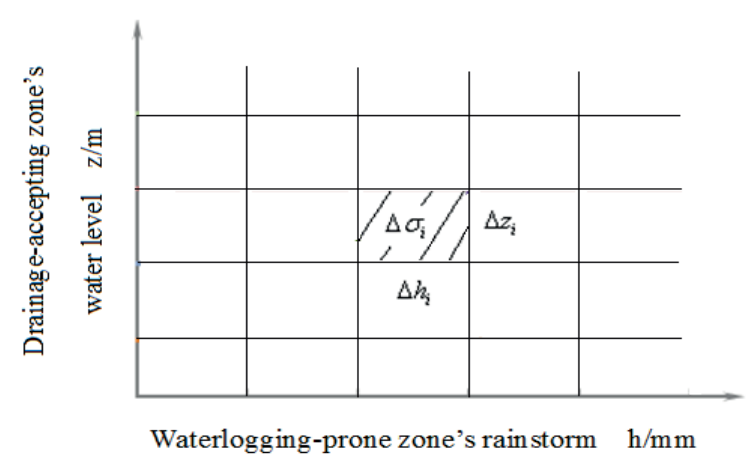

Fig. 1 Sketch map of division of integral area.

The length and width of each small rectangle region $\Delta \sigma_{i}$ are $\Delta h_{i}$ and $\Delta z_{i}$, respectively. If the coordinate of the rectangle's lower left corner is $\left(h_{i}, z_{i}\right)$, the coordinates of the other three corners are $\left(h_{i}+\Delta h_{i}, z_{i}\right),\left(h_{i}, z_{i}+\Delta z_{i}\right)$ and $\left(h_{i}+\Delta h_{i}, z_{i}+\Delta z_{i}\right)$. If the joint distribution of the rainfall in a waterlog-prone zone, $H$, and the water level in a drainage-accepting zone, $Z$, is expressed as $F(h, z)$. Then:

$$
\Delta p_{i}=\iint_{\Delta \sigma_{i}} f(h, z) \mathrm{d} h \mathrm{~d} z=\left.F(h, z)\right|_{\Delta \sigma_{i}}=F\left(h_{i}+\Delta h_{i}, z_{i}+\Delta z_{i}\right)-F\left(h_{i}, z_{i}+\Delta z_{i}\right)-F\left(h_{i}+\Delta h_{i}, z_{i}\right)+F\left(h_{i}, z_{i}\right)
$$


The average waterlogging loss, $\overline{s_{j i}}$, at $\Delta \sigma_{i}$ can be calculated with the following equation:

$$
\overline{s_{j i}}=g_{j}\left(h_{i}+\frac{1}{2} \Delta h_{i}, z_{i}+\frac{1}{2} \Delta z_{i}\right)
$$

Therefore, the key of waterlogging control benefit estimation is to calculate the join distribution, $F$ $\left(h_{i}, z_{i}\right)$ and the probability density, $f\left(h_{i}, z_{i}\right)$, and the waterlogging loss, $g_{i}\left(h_{i}, z_{i}\right)$.

\subsection{The steps of waterlogging control benefit estimation}

According to the analysis above, the waterlogging control benefit of a control project can be calculated in following steps:

(1) Collect and organize data and obtain the necessary relation curve in calculating waterlogging loss, combining with analysis and calculation.

(2) Collect data about the annual maximum rainstorms of the waterlog-prone zone and the water levels in the drainage-accepting zone, and analyse and determine the marginal distribution, the joint distribution and joint probability density function of the two variables.

(3) Determine the integral region according to the annual maximum rainstorms, marginal distribution of the corresponding water levels in the drainage-accepting zone and their joint probability density distribution. Rationally divide the integral region.

(4) Calculate the individual probability, $\Delta p_{i}$, of each segmented small rectangular region, and obtain the region's difference value of average waterlogging $\operatorname{loss} \overline{s_{1 i}}$, with a waterlogging control project and average $\operatorname{loss} \overline{S_{0 \mathrm{i}}}$, without a control project. The difference value is the region's waterlogging control benefit, $\Delta B$.

(5) Calculate the average waterlogging control benefit, $B \approx \sum_{i=1}^{n} \Delta B_{i} \Delta p_{i}$, i.e. the weighted average is obtained of all the waterlogging control benefits of all the small rectangle areas, using the probability as the weight. The average is approximate to the waterlogging control benefit of the waterlogging control project

\section{CASE ANALYSIS}

A waterlog-prone zone in the town of Yangshan of Guangdong Province was used as an example to briefly illustrate the analysis process of the waterlogging control benefit. In recent years, the waterlog-prone zone has developed so rapidly that original vegetable farming land has been changed to urban land. We only calculated the waterlogging loss and waterlogging control benefit in the first year (2010) after the waterlogging control project construction.

First, we obtained the spatial distribution of the various disaster-bearing bodies or diverse assets in the waterlogging-prone zone and got the relation of the submerging depth with the waterlogging loss rate, which uses submerging time as a parameter.

Table 1 shows the estimated values of various classified assets in the waterlogging-prone zone in 2010, simplified to the average distribution, and also includes the 2010 average waterlogging loss rate of each classified asset under different submerging depth.

Then, we analysed the marginal distribution and joint distribution of the variables, the rainstorm in the waterlog-prone zone and the water level in the drainage-accepting zone, to obtain the joint probability density function of the two variables.

The marginal distribution and marginal probability density function of the annual maximum daily rainstorm, $H$, are $F_{H}(h)$ and $f_{H}(h)$, respectively, and of the corresponding daily average water level, $Z$, are $F_{z}(z)$ and $f_{z}(\mathrm{z})$, respectively. We found that a P-III type distribution fits both the marginal distribution $F_{H}(h)$, and the corresponding daily average flood level, $F_{z}(z)$. The statistical parameters were $\bar{h}=97 \mathrm{~mm}, C_{v}=0.38, C_{s}=1.33$ and $\bar{z}=60.57 \mathrm{~m}, C_{v}=0.024$, and $C_{s}=0.63$, respectively. $f_{H}(h)$ and $f_{z}(z)$ are respectively shown as follows: 
Table 1 The value of each asset and the waterlog loss percentage in the case of different submerge in 2010.

\begin{tabular}{|c|c|c|c|c|c|}
\hline \multirow[t]{2}{*}{ Asset classification } & \multirow{2}{*}{$\begin{array}{l}\text { All kinds of } \\
\text { asset } \\
\text { value/100 } \\
\text { Yuan }\end{array}$} & \multicolumn{4}{|c|}{$\begin{array}{l}\text { The social-asset loss ratio under different } \\
\text { submerging depth }(\%)\end{array}$} \\
\hline & & $0 \sim 0.3 \mathrm{~m}$ & $0.3 \sim 0.6 \mathrm{~m}$ & $0.6 \sim 0.9 \mathrm{~m}$ & $0.9 \sim 1.2 \mathrm{~m}$ \\
\hline Fixed assets of industry and commerce & 0.36 & 2.5 & 5 & 5 & 5 \\
\hline Flow assets of industry and commerce & 0.10 & 2.5 & 6 & 8 & 10 \\
\hline $\begin{array}{l}\text { Business inventory goods and } \\
\text { materials }\end{array}$ & 0.09 & 1 & 2 & 3 & 4 \\
\hline Assets of construction industry & 0.15 & 0.5 & 1 & 2 & 3 \\
\hline The communication system & 0.05 & 0.5 & 1 & 2 & 3 \\
\hline Residential property & 0.18 & 1.5 & 4 & 8 & 10 \\
\hline Residential housing & 0.37 & 1 & 4 & 8 & 10 \\
\hline $\begin{array}{l}\text { Foundation engineering like traffic, } \\
\text { water supply, etc. }\end{array}$ & 0.57 & 0 & 1.5 & 3 & 5 \\
\hline Individual enterprises & 0.001 & 2.5 & 5 & 5 & 5 \\
\hline
\end{tabular}

$$
\begin{aligned}
& f_{H}(h)=\frac{0.0408^{2.2613}}{\Gamma(2.2613)}(h-41.57)^{1.2613} e^{-0.0408(h-41.57)}, \quad h \geq 41.57 \\
& f_{Z}(z)=\frac{2.1838^{10.0781}}{\Gamma(10.0781)}(z-55.96)^{9.0781} e^{-2.1838(z-55.96),}, z \geq 55.96
\end{aligned}
$$

The Clayton Copula function described the correlation structure between the maximum rainstorm and corresponding daily average water level better than a Gumbel Copula function. In this case, $\theta=10.5$. Consequently, we got the joint distribution, $F(h, z)$, and the joint probability density function, $f(h, \mathrm{z})$, respectively expressed as follows:

$$
\begin{aligned}
& F(h, z)=\left\{\left[F_{H}(h)\right]^{-10.5}+\left[F_{Z}(z)\right]^{-10.5}-1\right\}^{-1 / 10.5} \\
& f(h, z)=11.5\left[F_{H}(h) F_{Z}(z)\right]^{-11.5}\left\{\left[F_{H}(h)\right]^{-10.5}+\left[F_{Z}(z)\right]^{-10.5}-1\right\}^{\frac{-1}{10.5}-2} f_{H}(h) f_{Z}(z)
\end{aligned}
$$

Based on the joint probability density distribution (Fig. 2) and the marginal distribution, the integral area was defined as $(41.57 \sim 200 \mathrm{~mm}, 55.96 \sim 64.5 \mathrm{~m})$. The waterlogging design combination of the waterlogging-prone zone was between the 1-in-10 years rainstorm $(146 \mathrm{~mm})$ and the 1-in-5 years annual highest daily average water level $(62.21 \mathrm{~m})$ in the drainage-accepting zone. Hence, the integral region was segmented as shown in Fig. 3, integrating the waterlogging control design combination.

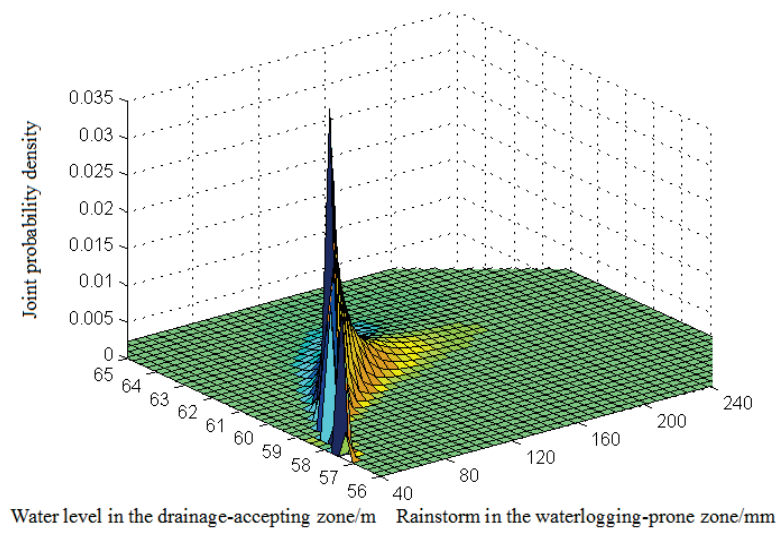

Fig. 2 The joint probability density distribution maps of the two variables. 


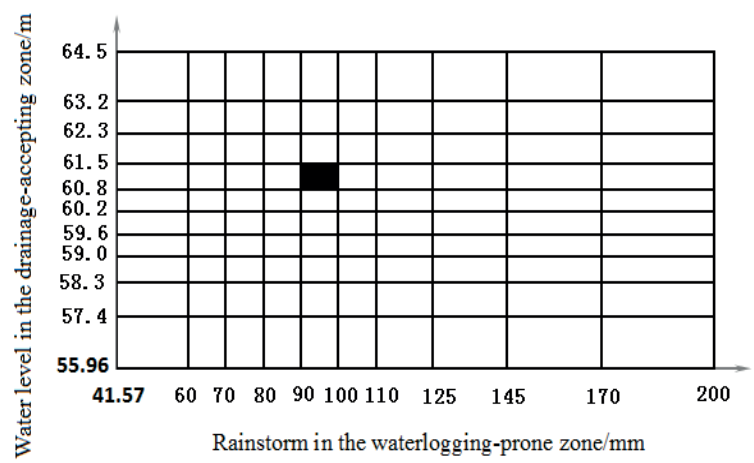

Fig. 3 The map of division of integral area.

Then, each region was calculated, and the region in Fig. 3 was used as an example to illuminate the analysis.

The midpoint coordinates of the area were $(95,61.15)$, suggesting the rainstorm of the waterlogging-prone zone was $95 \mathrm{~mm}$ and the corresponding water level was $61.15 \mathrm{~m}$. Because the design rainstorm and the waterlog control design water level were $146 \mathrm{~mm}$ and $62.21 \mathrm{~m}$, respectively, the area had no waterlogging loss with the waterlogging control project. So, the water-logging control benefit of this region was expressed as: $\Delta B_{i}=\overline{s_{0 i}}-\overline{s_{1 i}}=163.64-0=1.64$ million.

The four vertex coordinates of this area were $(90,60.8),(100,60.8),(100,61.5)$ and $(90$, $61.5)$, respectively. So the frequency of the area was $\Delta p_{i}=F(100,61.5)-F(100,60.8)-F(90$, $61.5)+F(90,60.8)=0.6127-0.5708-0.5102+0.5030=0.0347$.

Similarly, the waterlogging control benefit, $\Delta B_{i}$, and probability weight, $\Delta p_{i}$, of each area could be obtained, and therefore the 2010 waterlogging control benefit was calculated as $B=\sum \Delta B_{i} \Delta p_{i}=0.695$ (million Yuan).

\section{CONCLUSION}

None of the existing estimation methods of the direct benefit of waterlogging control projects is suitable for the changing environment. The estimation model of waterlogging control benefits proposed in this paper overcomes this defect. It is suitable for the changes of both disasterinducing environment and disaster-bearing bodies. In addition, it considers all the combinations between rainstorms of the waterlogging-prone zone and water levels in the drainage-accepting zone. Therefore, the proposed estimation method of waterlogging control benefits can objectively reflect the actual benefits, and thus provides a scientific basis for the rational decisions on waterlogging control project construction. But the application of the model will usually be restricted due to basic data deficiencies.

Acknowledgements The authors would like to thank the Open Foundation of State Key Laboratory of Hydraulics and Mountain River Engineering for support the research (Project 1113).

\section{REFERENCES}

Guo Qi (2002) Analysis and calculation on benefit of water project in lightening water logging disaster. Journal of Yangtze River Scientific Research Institute 19(6), 56-58.

Jia Run-fu and Tian Xiaojuan (2005) Research on the method for analyzing the benefit of waterlogging resistant engineering and its application. Water Conservancy Science and Technology and Economy 11(8), 454-456.

Li Jiren, Ding Zhixiong and Huang Shifeng (2003) Research of flood and waterlogging loss assessment model based on spatial distribution social-economic database. Journal of China Institute of Water 1(2), 104-110.

Liu Zengmei and Chen Zhishen (2009) Risk study of the bivariate encounter of interzone rainstorm and flood level of the outer river. Advances in Water Science 20(5), 619-625.

Liu Zengmei and Chen Zhishen. (2011) Study on the standard of waterlog prevention based on two waterlog-inducing factors. Journal of Hydroelectric Engineering 30(3), 44-50.

Shi Xichan and Jiang Shuixin. (2005) Water conservancy engineering economy. Chinese Water Conservancy and Water Power Publisher 2005, 134-139. 
Wang Li-ping, Wang Xiugui and Gao Shichun (2008) Water conservancy engineering economy. Chinese Water Conservancy and Water Power Publisher 2008, 145-157.

Zhang Dazhi (2004)The methods of calculation on the benefit of manage waterlogging. Guangdong Water Resources and Hydropower (1), 28-30.

Zhu Xuping, Liao Xinyu and Tang Deshan. (2007) Analysis and evaluation of waterlog control benefits in Liaohe River Basin flood control system. Water Power 33(3), 22-25. 